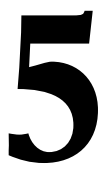

\title{
EDUCACIÓN SUPERIOR EN CONTEXTOS DE CUASI MERCADOS
}

\section{(HIGHER EDUCATION IN CONTEXTS OF QUASI MARKETS)}

Camilo Wee

Héctor Monarca

Universidad Autónoma de Madrid

DOI: $10.5944 / e d u c X X 1.20047$

Cómo referenciar este artículo/How to reference this article:

Wee, C. y Monarca, H. (2019). Educación Superior en contextos de cuasi mercados. Educación XX1, 22(1), 117-138, doi: 10.5944/educXX1.20047

Wee, C. \& Monarca, H. (2019). Educación superior en contextos de cuasi mercados. [Higher Education in contexts of quasi markets]. Educación XX1, 22(1), 117-138, doi: 10.5944/ educXX1.20047

\section{RESUMEN}

La Universidad debe ser comprendida en el marco de los procesos sociohistóricos que le dieron origen y las condiciones actuales que la exponen a demandas, presiones y tensiones diversas. Desde esta perspectiva, el presente trabajo pretende poner en debate la relación entre la Educación superior y el mercado, haciendo hincapié en la influencia que esta relación tiene en la configuración y resignificación de las funciones que la Universidad asume: docencia, investigación y extensión. De esta manera, se pretende analizar los efectos que las diversas posturas tienen en relación con estas funciones; las cuales se mueven entre tendencias democratizadoras de los espacios públicos y el acceso al conocimiento y otras que promueven la mercantilización de la educación superior. A partir de este análisis se hace alusión a diferentes modelos, los cuales reflejan diversas formas de apropiación y concreción de las funciones de la Universidad en un contexto de cuasi mercado, que ejerce presiones específicas en la configuración de las mismas. En definitiva, se 
concluye que la Universidad ha tomado un Modelo esencialmente funcional, que la vincula de manera preferente al mundo laboral y el mercado de una forma lineal, mercantilista y acrítica; alejándola cada vez más de sus funciones clásicas que la relacionan a la transmisión crítica del conocimiento científico y la formación cultural y humana superior.

\section{PALABRAS CLAVE}

Educación superior; organización y gestión; cambio organizacional; nueva gestión pública; cuasi mercado.

\section{ABSTRACT}

The University must be understood under the framework of the sociohistorical processes of its origin and the current conditions that expose it to claims, pressures and tensions. From this perspective, the present work aims to debate the relationship between higher education and the market, highlighting the influence that this alliance has in the setting and resignificance of the functions that the university embraces: teaching, research and extension. This way, the aim would be to analyze the effect that the diverse postures have in relation to these functions, which move between democratic trends of public spaces, access to knowledge and others that promote the commercialization of higher education. Considering the former analysis, different models have been mentioned which reflect several ways of appropriation and specification of the functions of university in the context of quasi market, causing specific pressures on the setting of the conditions previously stated. Finally, it is concluded that the university has taken an essentially functional model linked preferably to the labor world and the market in a linear, mercantilist and acritical format; causing a systematic separation of its classic role of critical transmission of scientific knowledge and the cultural and human formation.

\section{KEY WORDS}

Higher education; organization and management; organizational change; new public management; quasi market. 


\section{INTRODUCCIÓN}

La acción de gobernar de los Estados según Veiga-Neto (2010) se ha visto afectada por diversos poderes e intereses que han redefinido el deber ser de los gobiernos, escenario que parece imponerle nuevas funciones a la Universidad frente a una aparente ecuación costo-beneficio.

En este sentido, como lo menciona Herrera (2013), analizar la relación entre la Universidad y los modelos económicos, demuestra cómo esta afecta en la autonomía de la educación superior frente a las nuevas lógicas mercantilistas. Situación que queda en evidencia al analizar la racionalidad de las ciencias económicas fundamentada en las políticas educativas.

En esta línea, Foucault (2008) plantea la premisa de que el mercado actúa como instrumento regulador y de limitación de las acciones del Estado a través de una economía política que relaciona el poder económico y el poder político. Lo cual, desde el análisis de la teoría del capital humano, demuestra que la Universidad y sus funciones se proponen como una inversión que conlleva costos y oportunidades (Herrera, 2013).

En este contexto, Massé (2008) apunta a que la Universidad vive un proceso de descomposición institucional ligado a la lógica general del capitalismo y la globalización, que condiciona de diversas maneras el quehacer y las funciones de la Universidad. En este proceso, se pueden identificar iniciativas utilizadas como mecanismos de presión en la relación Universidad-Estado, destacando de entre ellos la "rendición de cuentas», asociada en este caso a la reducción del gasto público en educación (Pedroza, 2005).

Desde esta perspectiva, el texto analiza la influencia que ejerce el mercado en las funciones de la Universidad, pretendiendo poner en debate la relación Universidad-Estado-Mercado, desde un análisis histórico de sus funciones, asumiendo así que la Universidad no es algo atemporal ni neutro; sino una construcción socio-histórica (Medina, 2005). Este análisis, contribuirá a reflexionar acerca de los diversos modelos de Universidad que en la actualidad emergen y se consolidan a nivel internacional ante este nuevo escenario.

Por tanto, se pretende contrastar dos fuerzas antagonistas que se pueden reconocer en la universidad de hoy, las cuales, según Martinetto (2008), pueden descubrir los elementos constitutivos de los posibles y diferentes modelos universitarios propuestos y en pugna por convertirse en el modelo hegemónico a instaurar. Por un lado, un modelo con tendencia a democratizar los espacios públicos y el conocimiento libre para el bien 
común, por otro, un modelo de mercantilización de la educación y el conocimiento (Juarros y Nairdof, 2007). Entre ambos extremos, tal vez, una gran variedad de alternativas.

\section{PERSPECTIVA HISTÓRICA DE LA UNIVERSIDAD}

Para abordar el debate relacionado con la Universidad y el actual contexto mercantilista en el que se encuentra inserta es necesario comenzar por un análisis histórico, debido a que el concepto de Universidad ha cambiado sustancialmente a lo largo del tiempo (Huanca, 2004).

De esta forma, Tünnermann (2003) identifica el surgimiento de la Universidad tal como la conocemos, en la época medieval, a mediados del siglo XII; aunque reconoce que su apogeo se produjo en siglos posteriores. Asimismo, Geuna (1999) realiza una descripción de los diferentes periodos de evolución de la Universidad, distinguiendo cinco fases: 1) Nacimiento de la Universidad, desde finales del siglo XII hasta comienzos del siglo XVI, 2) El período de decadencia, entre la segunda mitad del siglo XVI y finales del siglo XVIII, 3) La transformación alemana, desde comienzos del siglo XIX hasta la Segunda Guerra Mundial, 4) La expansión y diversificación, desde 1945 hasta finales de 1970, y 5) La contribución al desarrollo económico, desde 1980 hasta comienzos del siglo XXI.

Por su parte, Mora (2004) reconoce que la actual Universidad nace a principio del siglo XIX, cuando se salta de la Universidad medieval a la Universidad moderna, dando paso a tres grandes modelos en los que las instituciones de educación superior de la época basarían su funcionamiento:

- El modelo alemán, heredado del idealismo alemán del siglo XVIII, el cual se sustentaba en la idea de que una sociedad con personas formadas científicamente sería capaz de generar avances a nivel social, cultural y económico. Fue organizado por instituciones públicas, con docentes establecidos como funcionarios públicos, en donde el objetivo principal de la Universidad era generar conocimiento científico.

- El modelo francés, en el cual las Universidades pasaron a ser parte de la administración del Estado para formar a los profesionales que el mismo Estado necesitaba, haciendo hincapié en que las instituciones estarían al servicio del Estado más que al de la sociedad. En este caso, se reconoce como su objetivo principal el formar a los profesionales que el Estado-nación organizado por la Francia napoleónica necesitaba; el cual fue exportado a otros países del sur de Europa. 
- El modelo anglosajón, dicho modelo mantuvo el estatuto de instituciones privadas que todas las universidades europeas tuvieron hasta principios del siglo XIX. El cual vivió su apogeo en las universidades británicas, siendo extendido a Norteamérica, presentando como objetivo principal la premisa que personas bien formadas serían capaces de servir adecuadamente a las necesidades de las nuevas empresas o las del propio Estado. Además, el modelo anglosajón destaca debido a que resistió de mejor forma el paso del tiempo, adaptándose rápidamente al contexto actual.

Por otra parte, C. García (2008) reconoce 5 estadios evolutivos por los cuales la Universidad moderna ha llegado a ser la institución con las funciones que se le conoce hoy en día, destacando de entre ellos los siguientes:

- La década de los 50, conocida como «La edad de la inocencia», denominación que se le atribuye debido al optimismo que despertaba la educación como garantía de mayor productividad económica y movilidad social.

- La década del 60, conocida como "La edad del escepticismo», nombre que se le designa debido a que en esos años se develaron, por un lado, la imposibilidad de lograr desarrollos armónicos de las sociedades bajo los esquemas desarrollistas, al igual que se demostró el carácter inequitativo de la expansión de los sistemas educativos.

- En la década de los 80, donde surgen con mayor fuerza los enfoques de la acción participativa, cuyo principal objetivo era la necesidad de mayor relación con el entorno social, tanto en la producción como en la distribución de conocimientos.

- En la década de los 90, época en la que se comienza a revalorizar la subjetividad social y la dimensión cualitativa de la vida social; los actores sociales y la sociedad civil; entendiendo la democracia como expansión de la conciencia reflexiva en todos los espacios de la interacción social. De la misma forma, esta época es reconocida por la generación de nuevas reformas que apuntaron fundamentalmente a la implantación de sistemas de evaluación y acreditación; con el objetivo de fortalecer las relaciones con el sector productivo, y a impulsar nuevos modelos de financiamiento.

- En la presente década, surge el énfasis de la necesidad de afianzar el compromiso social de la universidad con un desarrollo humano y sustentable, de esta forma se pretende hacer frente a la globalización; proceso que se considera ha acentuado las desigualdades entre los países. 
Dicho esto, existen autores que postulan que la Universidad moderna y el mercado están relacionados desde hace muchos años, como es el caso de Brunner (2008a), quien indica que desde el siglo XII las universidades se han desarrollado en un entorno que si bien no puede llamarse propiamente de mercado ya reunía algunos de los rasgos fundamentales de este.

Por otra parte, en el estudio de la relación Universidad-Mercado y en el contexto de la Universidad moderna, el autor recién mencionado destaca lo aportado por Adam Smith, quien desarrolló dos grandes líneas de análisis de esta temática: una asociada con la teoría del capital humano y otra con el análisis del comportamiento económico de las universidades.

Referido a la Teoría del capital humano, Smith (1976) realiza una comparación entre hombres y máquinas, afirmando que «Cuando se emplea cualquier máquina costosa debe suponerse que el servicio extraordinario que prestará durante su vida útil compensará el capital invertido en ella y producirá al menos una utilidad o ganancia para su dueño» (p. 113). En este sentido, Bowman (1972) reconoce que dicha comparación entre hombres y máquinas se considera como el antecedente principal para el desarrollo de la teoría del capital humano, lo que queda demostrado en afirmaciones realizadas por el propio Smith (1976), señalando que el trabajo que un hombre aprende a realizar debe compensar en su totalidad el gasto incurrido en su educación, el que debe generar al menos una ganancia similar al capital invertido. En esta línea, Brunner (2008a) menciona que lo aportado por Smith se traduce en que las inversiones en educación que mejoran la capacidad de una persona para realizar un trabajo productivo debe generar un retorno, calificado como un rendimiento de la inversión realizada en los seres humanos.

Por otro lado, referido al comportamiento económico de las universidades, Smith (1976) incluye en su análisis el argumento del bien público, no obstante, sostiene que las Universidades pueden tener un ingreso suficiente para costear sus gastos si los alumnos y alumnas pagan un arancel, recurriendo al financiamiento público solo subsidiariamente, aunque no exento de riesgos. Por lo que, en lo relacionado con la fuente de financiamiento de la Universidad, Brunner (2008a) señala que en ello se encuentra el núcleo del argumento smithsiano, pues si la retribución de los profesores no proviene del pago producido por los estudiantes, y proviniese únicamente del patrimonio público, sería imposible obtener un servicio de calidad relevante para la sociedad.

Esto se argumenta al señalar que el ingreso de los maestros conduce a un servicio educativo de calidad únicamente si es obtenido en un contexto de libre competencia y rivalidad entre competidores, comprometiendo a 
los docentes por desalojar unos a otros del empleo, obligando a cada uno a ejercer su trabajo con la máxima exactitud (Smith, 1976).

\section{UNIVERSIDAD, SOCIEDAD, ESTADO Y MERCADO}

En la evolución histórica de la Universidad, su concepción como un bien de mercado destaca como una de las principales fuentes en el cambio de sus funciones (Herrera 2013), causando que muchas Universidades se hayan visto forzadas a implementar un modelo económico. Dicha perspectiva, atenta contra los postulados que afirman que la educación debe ser entendida como un bien público y social que debe ser sostenido prioritariamente por los Estados (Corti, Oliva y De la Cruz, 2015).

En este sentido, a partir de la premisa planteada por Dias (2008), quien afirma que la educación es un bien público, derecho de todos, deber del Estado; se puede apreciar una vinculación entre educación y sociedad, desde el protagonismo que la educación posee en la construcción de esta.

En vista de esta relación, se cree que el Estado debería poder regular el funcionamiento, acceso y financiamiento que acerque a las personas con la Universidad, así como lo menciona Corti et. al. (2015) al resaltar la concepción de la educación como un bien público y social que debe ser sostenido prioritariamente por los Estados, de manera que contribuya a la creación de una conciencia solidaria y comprometida con el logro de mayores niveles de inclusión social y educativa.

Sin embargo, esto parece ser una realidad cada vez más lejana, debido a las nuevas fuerzas que, según Newman (2000), están remodelando la educación superior, haciendo referencia a cambios impulsados por el ingreso de nuevos proveedores que han modificado el entorno de la educación superior con una aparente visión competitiva. A cambio de una mayor injerencia del mercado, marcando una tendencia que perturba la tranquilidad de un sistema que hasta hace años se consideraba estable y confiado en sí mismo.

En cualquier caso, desde su origen, la Universidad se ha conformado en un espacio de tensiones y conflictos diversos relacionados con sus funciones, demandas y gobernanzas (Monarca, 2014). Ahora bien, en la actualidad, las funciones de la Universidad se reconocen a través de la docencia, investigación y extensión, siendo esta última la función designada para acercar a la Universidad con la sociedad. Tal como lo menciona C. García (2008, p. 132), «La función social de la universidad se ha ejercido y se sigue ejerciendo a través de la extensión universitaria». 
Sin embargo, como lo plantea Dias (2008), esto no parece ser suficiente, puesto que la universidad bajo la extensión universitaria parece únicamente ofrecer servicios a determinados sectores externos, creando puentes a la sociedad sin reflexionar sobre los significados de esas acciones. Por lo que la Universidad, en su rol formativo, debería repensar por entero su actuación con una perspectiva social.

Del mismo modo, Tünnermann (2013) se refiere a la relación Universidad-Sociedad a partir de sus funciones, ejercidas desde una perspectiva ética que contribuya a poner de manifiesto esa responsabilidad social de la que deben ser protagonistas todos los estamentos universitarios: directivos, administradores, docentes, investigadores y los estudiantes; con el fin último de hacer de la responsabilidad social el eje de la acción universitaria. Tal como lo indica Dias (2008, p. 13) «Lugar del ethos teórico y del ethos social, la universidad debe ser un espacio público de elaboración de un análisis crítico que supere el déficit de la globalización».

En un intento por presentar una Universidad comprometida con sus funciones sociales, C. García (2008) plantea que la extensión no debería ser una añadidura, sino que debería ser su razón de ser; extendiendo los servicios esenciales que la universidad genera, los cuales se reconocen como la producción de conocimientos ligada a la formación de profesionales a todos los sectores de la economía, tanto formal como informal, y a todos los grupos sociales.

Asimismo, la UNESCO desde la la Primera Conferencia Mundial sobre Educación Superior, celebrada en París en el año 1998, se refiere a la relación de la Universidad y la sociedad a través de la noción de pertinencia, la cual pretendía «ayudar a las mujeres y hombres, a las sociedades y a las comunidades de naciones a avanzar hacia un futuro mejor y un mundo más justo, más humano, más solidario y pacífico» (Mayor, 1998, p. 1), poniendo especial hincapié en la influencia que la Universidad tiene en el desarrollo de las sociedades.

Sin embargo, conceptualizar la vinculación entre sociedad y Universidad a través de la pertinencia no está exento de críticas, pues como lo señala Naidorf, Giordana y Horn (2007), la pertinencia como concepto se propone redefinir los vínculos entre Universidad y sociedad, lo que presupone una operación teórica que parece ser contradictoria, debido a que interpreta su relación como objetos aislables. Asimismo, dichos autores concluyen en que la pertinencia social de la Universidad es una categoría equívoca, debido a los siguientes argumentos: A) Desde el enfoque economicista, una institución cumple con parámetros de pertinencia social cuando responden a demandas del Mercado, es decir; su producción es eficiente en términos 
de aplicabilidad inmediata, son rentables, permiten obtener financiamiento externo, etc. B) Desde una perspectiva social, las actividades que realiza la Universidad son pertinentes cuando logran vincularse a la Sociedad de la cual forman parte y en la que contribuyen desde su especificidad al mejoramiento de las condiciones de vida de las mayorías, y lo hace de manera sustentable. C) Desde la categoría de pertinencia social, se busca redefinir la Universidad a partir de criterios de rendición de cuentas y de ajuste al Mercado, lo que impacta tanto en los aspectos académicos como en los financieros.

En contraste con los argumentos presentados, los cuales abogan por una estrecha relación Universidad-Estado y sus aparentes beneficios sociales, surgen desde el campo de la economía argumentos que de forma tajante refutan esta premisa. En este sentido, Friedman y Friedman (1980) plantean que «la educación superior produce beneficios sociales más allá y por encima de los beneficios que aprovechan los propios estudiantes» (p. 187), por lo que cuestionan la iniciativa de entregar subsidios a las instituciones de educación superior; asumiendo que no aseguran la mejora de la distribución de oportunidades económicas, por el contrario, favorece únicamente a aquellos jóvenes que acceden a este nivel educacional.

Es por esto que organizaciones como el Banco Mundial en su momento propusieron reducir el aporte público brindado a las Universidades, apoyando el cobro de aranceles a los alumnos y alumnas mediante créditos estudiantiles (The World Bank, 1988). Asimismo, Bloom, Hartley y Rosovsky (2006) postulan que en relación con el cálculo costo/beneficio aplicado a la educación superior, este produce mayores retornos privados que retornos sociales.

- Frente a una aparente dicotomía en la relación Estado-Mercado, surgen dos grandes modelos que parecen marcar tendencias en torno a las funciones que la Universidad cumple en la actualidad (Meléndez, Solís y Gómez, 2010), el modelo de universidad público y el modelo privado. En esta compleja relación, Lorente (2015) destaca importantes perspectivas de análisis que se generan en torno a la comprensión de diversas concepciones de la Universidad, destacando las siguientes: Perfil de las universidades privadas, Las universidades privadas presentan orientación docente y producen solo $3.35 \%$ de los artículos científicos, mientras que las públicas se orientan a las dos funciones esenciales de la universidad: docencia e investigación, y llevan a cabo el $96.65 \%$ de la producción científica en España (Conferencia de Rectores de las Universidades Españolas, 2015). 
- Financiación pública de lo privado: Becas y financiación autonómica, La existencia de becas públicas para cursar estudios en Universidades privadas, considerando que en las Universidades públicas existe la misma oferta. Lo que se considera una transferencia injustificada de fondos públicos al sector privado.

- Encarecimiento de la Universidad pública y reducción de la financiación pública, a través de recortes de financiamiento público y el aumento en las tasas a cobrar por los estudiantes.

De esta manera, se considera la irrupción de un modelo de Universidad neoliberal como un "caballo de troya», que parece estar generando una suerte de desmantelamiento de uno de los pilares del Estado de Bienestar, como es la educación en su nivel universitario (Lorente, 2015), a través de una emergente «privatización encubierta» que en el contexto europeo se ha llevado a cabo mediante la configuración del Espacio Europeo de Educación Superior EEES y el proceso de Bolonia; con las presentes connotaciones de privatización que en él se aprecian (Ferrer, 2009).

Frente a esta confusa relación entre lo público y lo privado es trascendental conceptualizar el significado de la Universidad pública, que a partir de lo aportado por Tamayo (2007) se puede definir como «El ejercicio libre de la razón, el privilegio de la escritura y la lectura, la unión dialéctica entre teoría y práctica, la argumentación razonada y la autorregulación autónoma, lo que llamamos «una comunidad académica» (p. 299).

\section{PERSPECTIVA INTERNACIONAL DE LA MERCANTILIZACIÓN DE LA EDUCACIÓN SUPERIOR}

Juarros y Nairdof (2007) mencionan que la Universidad se encuentra interpelada por diferentes requerimientos, provenientes tanto del sector privado como del Estado, los cuales condicionan sus estructuras organizativas y su producción de conocimiento. Debido a esto, uno de los grandes conflictos a resolver por parte de los gobiernos y las Universidades es cómo lograr acuerdos entre ambos sectores, considerando de esta forma al sector público, al sector privado y a la comunidad académica.

A raíz de esto nacen diferentes concepciones de cómo dirimir dicho conflicto, dando origen a dos grandes discursos antagónicos, uno que aboga por democratizar los espacios públicos y el conocimiento libre, y otro que promueve la mercantilización de la educación y el conocimiento. Lo que impacta tanto en las prácticas como en las formas de organización de cada Universidad, produciendo resultados diferentes en cada una 
de ellas, en función de las historias particulares que las determinan y, fundamentalmente, de su composición de fuerzas (Juarros y Nairdof, 2007).

En esta línea, existen diversos estudios que analizan el proceso por el cual las Universidades se han ido acomodando o resistiendo a las exigencias de un modelo mercantilista, los cuales han sido realizados en diferentes contextos (Brunner, 2008a; 2008b; Burston, 2017; D. García, 2013; Hartmann, 2017; Hayes y Findlow, 2017; Herrera, 2013; Ibarra, 2012; Richmond, 2006a; 2006b; Suárez, 2013).Uno de los casos en donde se puede observar la relación entre lo público y lo privado, y la acomodación o resistencia a las políticas mercantilistas es el caso del modelo Universitario británico, el cual según lo expuesto por M. García (2001) desde sus inicios se posicionó como uno de los modelos universitarios con mayor autonomía y fue el referente de países como España, Francia y Alemania. No obstante, a raíz de sucesos de índole político y económico se vio afectado por presiones que obligaron a reorganizar sus principios que hasta comienzos de los años 80 parecían inamovibles.

Por otro lado, como país precursor de instaurar rápidamente un modelo mercantilista de Universidad, destaca, entre otros, el caso mexicano, el cual no está exento de críticas, pues como bien señala Sirvanci (2004), para que el sistema educativo pueda ser reconocido como un mercado, se necesitaría que los estudiantes considerados consumidores, tuviesen libertad de elección frente a la casa de estudios a la que deseasen acceder; pero que en realidad no la tienen. En este contexto, Suárez (2013) menciona la gran cantidad de jóvenes que buscan matricularse en las instituciones públicas de prestigio, superando el límite de plazas ofertadas y generando un escenario excluyente que inclusive lleva a los jóvenes a abandonar sus estudios.

Frente a este escenario, Suárez (2013) en su investigación menciona que los estudiantes mexicanos se ven enfrentados a repartir sus matrículas dentro de las ofertas que el mercado universitario les ofrece, lo que a partir de datos obtenidos por la Encuesta Nacional de Alumnos de Educación Superior (ENAES), deja en evidencia problemáticas que se adhieren a este modelo de Universidad; tanto para estudiantes que poseen los medios económicos como los que no, debiendo optar por instituciones privadas que no satisfacen plenamente sus intereses. Además, para que el mercado de la educación superior se mantenga, necesita dinero, lo que se espera sea financiado con fondos públicos, al igual que por parte de los mismos estudiantes.

No obstante, una alta proporción de los estudiantes de México, como de muchos otros países, está compuesta por jóvenes provenientes de familias pobres, lo que representa un problema para quienes esperan lucrarse con 
este tipo de «clientes». Sin embargo, Organismos Internacionales como la Organización para la Cooperación y el Desarrollo Económicos (OCDE) sugieren a los gobiernos que implanten programas de créditos educativos, conocidos en la actualidad como becas y créditos (Suárez, 2013).

Asimismo, otro país que destaca por el desarrollo de un modelo mercantilista de Universidad es el caso chileno, el cual posee una economía política que lo asemeja a países como Corea, Japón e Indonesia, reconocidos por poseer un sistema universitario con clara orientación a la privatización. Chile se caracteriza por tener un sistema muy diverso (Brunner, 2008b), compuesto por 209 instituciones: 61 universidades, 43 institutos profesionales y 105 centros de formación técnica.

En este sentido, Brunner (2008b) caracteriza a los modelos con orientación a la privatización, a partir de las siguientes características: A) Existe un mercado de consumidores, el cual, en el caso chileno, se da de forma más visible en la enseñanza de grado, con una amplia libertad de elección para los estudiantes que pueden pagar por el servicio, ya sea directamente o a través de becas y créditos financiados por el gobierno, a partir del sistema financiero con aval del Estado o por las propias instituciones. B) Existe un mercado laboral de posiciones académicas, en el cual, en el caso de Chile, las instituciones estatales contratan y despiden a sus profesores, fijan su escala salarial y regulan, cada una en sus propios estatutos de la carrera del personal académico (Bernasconi y Rojas, 2003). C) Existe un sistema de mercado de prestigios institucionales, el cual es utilizado como señal de calidad; la atracción «de los mejores estudiantes y profesores» que, como bien lo señala Clark (1983), se conoce como la moneda de intercambio entre las Universidades.

Estas características se pueden apreciar en diversos contextos, además de los antes mencionados, destacan los cambios ocurridos en el sistema de educación superior argentino, el cual se ha visto impactado por una serie de nuevas políticas que pretenden modificar sus dos subsistemas de educación superior; constituidos por las Universidades y los institutos de educación superior no universitarios (Richmond, 2006a). Asimismo, esto se ha visto confirmado a partir del análisis de organismos internacionales, en este caso el Banco Mundial, quienes diagnosticaron que los principales problemas del sector Universitario argentino, radicaban en su impresionante crecimiento, en el mantenimiento de la gratuidad, la insuficiencia del gasto y en no haber limitado el ingreso (Paviglianiti, Nosiglia y Márquina, 1996).

Por tanto, el Banco mundial, propone desarrollar instituciones de educación no universitarias, públicas y privadas, de tipo politécnico, ciclos cortos, institutos técnicos, colegios comunitarios, educación a distancia 
y programas de enseñanza abierta, enfatizando en que estas opciones se realizan con programas de bajo costo y permiten ofrecer capacitación que se adhiere a la demanda del mercado, los cuales pueden ser relacionados con programas universitarios (Paviglianiti et. al., 1996).

Frente a este escenario, Richmond (2006a) menciona que las políticas nacionales de articulación de los dos subsistemas de la educación superior Argentina se presentan como una estrategia orientada a transferir de forma encubierta el financiamiento de la educación superior a las provincias y a los ciudadanos, convirtiéndose en un bien de consumo al que tendrán acceso quienes puedan pagar; redefiniendo la relación Estado-UniversidadSociedad.

En esta línea, Herrera (2013) menciona que de igual forma el modelo de Universidad colombiano está fuertemente influido por políticas académicas que esconden un trasfondo económico que poco a poco convierte los servicios educativos en una fábrica de la nueva economía del conocimiento, puesto que las políticas educativas en este sector han obedecido más a criterios fiscalistas que a los criterios académicos. Además, critica la meritocracia existente en la educación superior, resaltando el hecho de ser usado como mecanismo equitativo de reparto de las escasas oportunidades que se brindan a la sociedad; cuando lo esperable es que se aumenten dichas oportunidades con mayor presencia del Estado en el suministro de los servicios.

Por el contrario, fuera del continente americano se reconoce al modelo de Universidad alemán como uno de los más resistentes a las demandas del mercado neoliberal, como bien lo indica M. García (2001, p. 326), «Los presupuestos que históricamente han guiado a este modelo universitario revelan una concepción integrada de la educación, la sabiduría y el Estado», forjando una Universidad que articula la docencia, la investigación, el conocimiento, la libertad y la autonomía (Rau, 1993).

Además, M. García (2001) destaca una serie de hitos que evidencian dicha resistencia: A) La creación de las Fachhochschulen, instituciones homólogas a las politécnicas británicas, las cuales nacen del ascenso de las antiguas escuelas profesionales de nivel inferior Fachschulen, convertidas en escuelas profesionales de nivel superior. La cual destaca por su orientación curricular e investigadora vinculada a las necesidades de la industria y del mercado laboral. B) La creación de las Gesamthochschule, una escuela superior total creada en la década de los setenta, con la función de proporcionar diversos tipos de educación y enseñanza terciaria flexible y adaptada a un abanico heterogéneo de estudiantes. C) El carácter inclusivo de la educación superior alemana, a partir de la función del Colle, institución 
de educación superior creada para la formación y la emisión del Abatir para estudiantes que independiente de su edad y circunstancias, desean ingresar a la Universidad; lo que representa el reconocimiento y la práctica de la igualdad de oportunidades en un sistema sumamente diverso en todos sus niveles.

Por otra parte, C. García (2005) considera que a partir del fenómeno de la globalización la Universidad está siendo afectada por un singular proceso, el de la internacionalización de la Universidad. Lo que en realidad no es algo totalmente nuevo, y que tiene como uno de sus mayores exponentes el ejemplo de Erasmo de Rotterdam y su extensa experiencia en movilidad académica durante el siglo XV, el cual inspiró a los líderes europeos a la creación del programa más grande de movilidad de estudiantes «ERASMUS» (Solanas, 2013). En cualquier caso, la internacionalización de la Universidad parece ser uno de los cambios más significativos de la Universidad contemporánea.

En esta línea, Araujo (2007) sostiene que los gobiernos han debido realizar grandes cambios que han afectado a la Universidad de forma directa, como es el caso de la reestructuración de la administración pública, que se reconoce como el paradigma del «nuevo gerenciamiento público» o «new public management»; que se presume aumenta las condiciones para implantar el fenómeno de la internacionalización de la Universidad, también interpretado como un proceso de expansión o globalización del neoliberalismo (Monarca, 2014).

A su vez, producto de la internacionalización, el flujo de intercambio de estudiantes se ha acrecentado notoriamente, tal como lo ha indicado la Organización para la Cooperación y el Desarrollo Económico, quienes afirman que a mediados de los noventa los países miembros de la OCDE se repartían el 85\% de los estudiantes extranjeros (OCDE, 2004). Además, señala que entre los años 2000 y 2011 la cantidad de estudiantes en formación en el extranjero ha presentado un crecimiento superior al $7 \%$ anual promedio, concentrado en países como Alemania, Australia, Canadá, Estados Unidos, Francia y Reino Unido (OCDE, 2013).

Por otro lado, la internacionalización en sus inicios argumentaba su creciente desarrollo aludiendo al fomento del intercambio académicoprofesional y la cooperación entre instituciones. Sin embargo, paulatinamente ha cambiado a un tipo de cooperación por competencia (De Wit, 2005), que se desarrolla en un mercado en el que las instituciones compiten por reclutar recursos humanos docentes, demostrando una aparente relación entre los procesos de internacionalización y de mercantilización (Solanas, 2013). 
De la misma manera, Tünnermann (2013) señala que se debe diferenciar claramente la internacionalización con la transnacionalización de la educación superior, debido a que esta última conlleva su transformación en un servicio sujeto a las reglas del mercado, con predominio de los intereses de las empresas educativas transnacionales. Mientras que en la internacionalización se debe propugnar por una cooperación internacional solidaria, basada en el diálogo intercultural y respetuosa de la idiosincrasia e identidad de los países participantes, así como por el diseño de redes interuniversitarias y de espacios académicos ampliados.

Por tanto, en la transnacionalización se intenta imponer el establecimiento en países menos desarrollados de filiales de universidades extranjeras, de promover una cooperación dominada por criterios asistenciales y de estimular la venta de franquicias académicas. Además, incluye la creación de universidades corporativas, auspiciadas por las grandes empresas transnacionales y las universidades virtuales, controladas por universidades y empresas de los países más desarrollados (Tünnermann, 2013).

A causa de estas y otras razones, tal como lo señala Robertson (2003), la Universidad parece estar perdiendo poco a poco su función social y comienza a integrarse rápidamente en una nueva racionalidad económica. El autor recién mencionado sostiene que en la actualidad la educación deja de servir a la comunidad para convertirse en un servicio que la sociedad adquiere. En consecuencia, como lo señala D. García (2013), parece necesario encarar los efectos de la globalización de la educación con la convicción de que la sociedad del conocimiento no es sinónimo del negocio de la inteligencia.

\section{CONCLUSIONES}

La Universidad debe ser comprendida en el marco de los procesos socio-históricos que le dieron origen y las condiciones actuales que la exponen a demandas, presiones y tensiones diversas, originadas a partir de requerimientos aparentemente sociales, que sin embargo se relacionan en forma directa al mundo laboral y del mercado; dando paso a un nuevo modelo universitario que se aleja de la idea de una Universidad concebida como un bien público y un derecho de todos.

Esto ha provocado que la Universidad pareciera estar perdiendo su orientación relacionada a sus funciones clásicas: docencia, investigación y extensión, las cuales apuntan al desarrollo de «la investigación científica, la transmisión crítica de la ciencia y la formación cultural y humana a nivel superior» (Medina, 2005, p. 19). 
Ante esto, se aprecia que existen diversas demandas y presiones que han llevado a la Universidad por este camino, desde los Organismos Internacionales, el poder político y el poder económico, que han logrado influir en los gobiernos y sus políticas. Por lo que se considera que la Universidad se encuentra interpelada por requerimientos provenientes tanto del sector privado como del Estado, condicionando la configuración de sus funciones; generando una particular relación entre lo privado y lo público guiada por una constante búsqueda de aumento de beneficios y disminución de pérdidas (Juarros y Nairdof, 2007).

Por otra parte, es necesario cuestionar el supuesto contexto de mercado presente en la educación superior, debido a que para que este pudiese ser considerado un escenario de mercado puro, los gobiernos deberían dejar de financiar a las instituciones y subsidiar a los estudiantes; generando en el mejor de los casos un contexto de cuasi mercado, y que de ninguna forma puede considerarse un mercado propiamente tal, a partir de estas condiciones (The National Center for Public Policy and Higher Education, 2003).

Producto de esto, las Universidades deben funcionar como si estuviesen en un real contexto de mercado, por lo que han debido extender sus actividades adaptándose a las reglas de la competencia. Fruto de este comportamiento, se pueden reconocer los contratos de desempeño con el gobierno y presupuestos regulados por las autoridades para la asignación de recursos para la docencia e investigación. Además de un aparente capitalismo académico, que ha visto sus orígenes en la necesidad de las instituciones por cubrir parte de sus gastos; mediante el cobro de aranceles, la comercialización de la investigación y la venta de servicios no tradicionales (Brunner, 2008a).

De esta manera, este contexto de cuasi mercado genera que las Universidades se comporten de forma similar a una empresa, operando como un capitalista de riesgo a modo de no perder sus ventajas competitivas, desarrollando políticas de precios como una estrategia de negocio; perjudicando en muchos casos sus funciones principales (Shattock, 2003).

Frente a esto, se reconoce que la Universidad ha modificado su modelo de formación para lograr dar respuesta a las necesidades de un nuevo contexto, caracterizado principalmente por la globalización, por la universalidad y las demandas de la sociedad del conocimiento (Mora, 2004). Sin embargo, esto ha provocado la irrupción de nuevos actores, que han facilitado a los estados la gestión de nuevos requerimientos sociales; sin que esto signifique un aumento en sus gastos públicos dirigidos a la Educación superior. 
En definitiva, la Educación superior es un derecho de todos, que actualmente se encuentra en una gran encrucijada, sucumbida por presiones provenientes de diferentes sectores que parecen desconfigurar sus principales funciones. Sin embargo, se debe procurar que esta situación sea regulada devolviendo mayor participación a los Estados, dejando de concebir a la Universidad como un servicio de mercado, y resaltando su compromiso con el logro de mayores niveles de inclusión social y educativa. 


\section{REFERENCIAS BIBLIOGRÁFICAS}

Araujo, S. (2007). Evaluación institucional y cambio universitario. Un difícil proceso de reconstrucción. En P. Krotsch, A. Camou y M. Prati (Coords.), Evaluando la evaluación: politicas universitarias, instituciones $y$ actores en Argentina y América Latina (pp. 69-94). Buenos Aires: Prometeo Libros.

Bernasconi, A. y Rojas, F. (2004). Informe sobre la educación superior en Chile: 1980-2003. Santiago de Chile: Editorial Universitaria.

Bloom, D., Hartley, M., \& Rosovsky, H. (2006). Beyond private gain: The public benefits of higher education. En J. Forest \& P. Altbach (Eds.), International Handbook of Higher Education (pp. 293-308). Dordrecht: Springer.

Bowman, M. (1972). Revolución en el pensamiento económico a causa del concepto de inversión humana. En M. Blaug (Eds), Economía de la Educación. Textos Escogidos (pp. 94122). Madrid: Tecnos.

Brunner, J. (2008a). Educación superior en Chile: Instituciones, mercados $y$ politicas gubernamentales, 19672007. [Tesis Doctoral]. Leiden: Leiden University.

Brunner, J. (2008b). El sistema de educación superior en Chile: Un enfoque de economía política comparada. Avaliação, 13(2), 451486.

Burston, M. (2017). A complex matter: charitable organization or corporate institution? A reflection on charity and its applicability in an era of marketdriven Higher Education in Australia, Critical Studies in Education, DOI: 10.1080/17508487.2017.1333520
Clark, B. (1983). The Higher Education SystemAcademic Organization in Cross-National Perspective. Berkeley, CA: Universiy of California Press.

Corti, A., Oliva, D. y De la Cruz, S. (2015). La internacionalización y el mercado universitario. Revista de la Educación Superior, $X \operatorname{LIV}(2)$, 47-60.

CRUE (2015): La universidad española en cifras. Año 2013 y curso académico 2013/2014. Madrid: CRUE.

De Wit, H. (2005). América Latina y Europa ante el fenómeno de la internacionalización. En J. Mora y N. Fernández. (Coords.), Educación superior, convergencia entre América Latina y Europa (pp. 222-226). Buenos Aires: Universidad Nacional de Tres de Febrero.

Dias, J. (2008). Calidad, pertinencia y responsabilidad social de la universidad latinoamericana $y$ caribeña. En A. Gazzola, y A. Didriksson, (Eds.), Tendencias de la Educación Superior en América Latina $y$ el Caribe (pp. 87-112). Caracas: IESALC-UNESCO.

Ferrer, J. (2009). Universidad: Servicio público frente a mercantilización. Revista Fuentes, 9, 27-41.

Friedman, M. y Friedman, R. (1980). Free to Choose. Orlando: Harcourt.

Foucault, M. (2008). Birth of biopolitics. Basingstoke: Palgrave Macmillan.

García, C. (2005). Complejidades de la globalización e internacionalización de la educación superior: Interrogantes para América Latina. Cuadernos del Cendes, 22(58), 1-22.

García, C. (2008). El compromiso social de las universidades. 
Conferencia central en el IX Congreso Iberoamericano de Extensión Universitaria Celebración de los 50 años de la Asociación Colombiana de Universidades. 25(67),129-134.

García, D. (2013). Aportaciones para el análisis de la cultura empresarial en la universidad mexicana: El caso del Tec de Monterrey. Revista Mexicana de Investigación Educativa, 18(56), 191221.

García, M. (2001). La universidad británica: ¿Un modelo para la universidad en otros países? Revista Española de Educación Comparada, 7,295-338.

García, R. (2012). Bioética en perspectiva latinoamericana. Revista Latinoamericana de Bioética, 12, 44-51.

Geuna, A. (1999). The economics of knowledge production: funding and the structure of university research. Northamptom, UK: Edward Elgar.

Hayes, A. y Findlow; S. (2017). The role of time in policymaking: a Bahraini model of higher education competition, Critical Studies in Education, DOI: 10.1080/17508487.2017.1358756

Hartmann, E. (2017). Quality assurance and the shift towards private governance in higher education: Europeanisation through the back door? Globalisation, Societies and Education, 15(3), 309-324, DOI: 10.1080/14767724.2017.1330138

Herrera, J. (2013). Ética, equidad y Meritocrácia en la mercantilización de la educación superior colombiana. Bioética, 13(1), 8-17.

Hermo, J., e Verger, A. (2010). Las políticas de convergencia de la educación superior: un estudio comparado entre el proceso de Bolonia y el Mercosur. Gestão Universitária na América Latina, 3(1), 1-16.

Huanca, R. (2004). La investigación universitaria de países en desarrollo y la visión de los académicos sobre la relación universidad empresa: Universidades públicas de la región occidental de Bolivia. [Tesis Doctoral]. Valencia: Universidad Politécnica de Valencia

Ibarra, E, (2012). Privatización y comercialización de la universidad: Las disputas por el conocimiento. Perfiles Educativos, XXXIV (número especial), 84-92.

Juarros, F., e Nairdof, J. (2007). Modelos universitarios en pugna: Democratización o mercantilización de la universidad y del conocimiento público en argentina. Revista da Avaliação da Educação Superior. 12(3), 483-504.

Lorente, R. (2015). El proceso de degradación de la Universidad y de su función pública en España. Arxius. 32, 139-160.

Martinetto, A. (2008). La década del 80 en la universidad pública. Normalización post-dictadura y transición hacia la reforma de los años 90. Valencia: Mimeo.

Massé, C. (2008). Autonomía estatal y universitaria, mercantilización del conocimiento y educación en el neoliberalismo. EDUGERE. Foro universitario, 12(41), 387-395.

Mayor, F. (1998). Prólogo. En UNESCO, Conferencia Mundial sobre la Educación Superior. La educación superior en el siglo XXI Visión y Acción (pp. 2-137). París: UNESCO.

Medina, R. (2005). Misiones y funciones de la universidad en el espacio europeo 
de educación superior. Revista española de pedagogía, 230, 17-42.

Meléndez, M., Sólís, P., y Gomez, J. (2010). Gobernanza y gestión de la universidad pública. Revista de Ciencias Sociales. XVI(2), 210-225.

Monarca (2014). Introducción. Evaluación de la calidad de la Educación Superior. En H. Monarca y J. Valle (Coords.), Evaluación de la Calidad de la Educación Superior en Iberoamérica (pp. 13-19). Madrid: GIPES-UAM.

Mora, J. (2004). La necesidad del cambio educativo para la sociedad del conocimiento. Revista Iberoamericana de Educación, 35, 13-37.

Naidorf, J., Giordana, P., y Horn, M. (2007). La pertinencia social de la Universidad como categoría equívoca. Nómadas, 27, 22-33

Newman, F. (2000). Saving Higher Education's Soul; The Futures Project. The Magazine of Higher Learning, 32(5), 16-23.

OCDE. (2004). Enseignement Supérieur: Internationalisation et commerce. París: Ediciones OCDE.

OCDE. (2013). Regards sur l'éducation 2013: Les indicateurs de l'OCDE. París: Ediciones OCDE.

Paviglianiti, N., Nosiglia, M., y Márquina, M. (1996). Recomposición neoconservadora. Lugar afectado: la universidad. Buenos Aires: Miño y Dávila.

Pedroza, F. (2005). La digitalización de la universidad en el capitalismo contemporáneo. En C. Massé (Coords.), La complejidad de las ciencias sociales en la sociedad de la información y la economía del conocimiento. México: Ed. El Colegio Mexiquense A.C.
Rau, E. (1993). Inertia and Resistance 10 Chunge of the Humboltian University. En C. Gellert (Eds.), Higher Education in Europe (pp. 37-38). London: Jessica Kingsley Pub.

Richmond, I. (2006a). Las políticas de mercado en el ámbito de la educación superior Argentina. Revista de Educación, 341, 757-779.

Richmond, I. (2006b). Las políticas de mercado en el ámbito de la educación superior Argentina: Políticas nacionales de articulación de los dos subsistemas de la educación superior. Revista Española de Educación Comparada, 12, 407-428.

Robertson, S. (2003). WTO/GATS and the global education services industry. Globalisation, Societies and Education, 1(3), 259-266.

Shattock, M. (2006). United Kingdom. En J. Forest, y P. Altbach (Eds.). International Handbook of Higher Education (pp.1019-1033). Dordrecht: Springer.

Sirvanci, B. (2004). Critical Issues for TQM Implementation in Higher Education. The TQM Magazine, 16(6), 382-386.

Smith, A. (1976). An Inquiry into the Nature and Causes of The Wealth of Nations. Chicago: The University of Chicago Press.

Solanas, F. (2013). Intercambio cooperativo versus mercantilización competitiva: Las políticas de movilidad académica en el Mercosur y la Unión Europea. Revista Iberoamericana de Educación Superior (Ries), 5(12), 3-22.

Suárez, M. (2013). Los estudiantes como consumidores Acercamiento a la mercantilización de la educación superior a través de las respuestas a la Encuesta Nacional de Alumnos de 
Educación Superior (ENAES). Perfiles Educativos, XXXV(139), 171-187.

Tamayo, A. (2007). En defensa de la Universidad Pública. Apuntes del CENES, 43(27), 293-304.

The National Center for Public Policy and Higher Education. (2003). Purposes, Policies, Performance in Higher Education and the Fulfillment of a State's Public Agenda. Disponible en http://bit.ly/2gEd1ye

The World Bank. (1988). Education in Sub-saharan Africa: Policies for Adjustment, Revitalization, and
Expansion. Washington, D.C.: The World Bank.

Tünnermann, C. (2003). La Universidad latinoamericana ante los retos del siglo XXI. México D.F.: Colección UDUAL.

Tünnermann, C. (2013). Diez respuestas de la educación superior a los desafíos contemporáneos. Universidades. LXIII(56), 5-14.

Veiga-Neto, A. (2010). Gubernamentalidad neoliberal: implicaciones para la educación. Revista Educación y Pedagogía, 22, 213-235. 


\section{PERFIL ACADÉMICO Y PROFESIONAL DE LOS AUTORES}

Camilo Patricio Wee Olivero. Universidad Autónoma de Madrid, Facultad de Formación de Profesorado y Educación, Departamento de Didáctica y Teoría de la Educación. Líneas de investigación: Política educativa.

Héctor Monarca. Universidad Autónoma de Madrid, Facultad de Formación de Profesorado y Educación, Departamento de Didáctica y Teoría de la Educación. Miembro del Grupo de Investigación sobre Políticas Educativas Supranacionales (GIPES). Líneas de investigación: política educativa, especialmente relacionadas con evaluaciones externas y desarrollo profesional docente.

Dirección de los autores: Universidad Autónoma de Madrid

C/ Tomás y Valiente, 3 28049 Cantoblanco (Madrid)

E-mail: camilo.wee@gmail.com hector.monarca@uam.es

Fecha recepción del artículo: 14. Octubre. 2017

Fecha modificación del artículo: 18. Marzo. 2018

Fecha aceptación del artículo: 21. Marzo. 2018

Fecha revisión para publicación: 20. Junio. 2018 